\title{
Preliminary Report on a Comparative Study on Patients' Satisfaction in Private and Public Hospital in the Perspective of Prevailing Health Management Constraints in Rajshahi
}

\author{
A R M Saifuddin Ekram ${ }^{1}$, M Fazlur Rahman ${ }^{2}$, S Abdullah Salim ${ }^{3}$, M Jawadul Haque ${ }^{4}$
}

\begin{abstract}
Assessment of patient satisfaction is required to help to improve health system performance and promote better governance of the hospital services. Despite resource constraints health personnel in private health care delivery centers are providing satisfactory health services than public hospitals. In the face of ever increasing demand for health care services strength, weakness and future prospects of private health care facilities should be evaluated and appraised. On the other hand health care delivery in public hospitals should be closely monitored for further improvement to meet the demands of people.
\end{abstract}

TAJ 2008; 21(1): 23-30

\section{Introduction}

Patient satisfaction has become an established indicator of the quality of health care yet, despite the abundance of measurement surveys reported in the literature, there are few theoretical underpinnings for this important concept. A review of the medical literature relating to the term "patient satisfaction" shows little research on the topic in the 1960s and 1970s. However, things began to pick up dramatically in the early 1980s. Between 1980 and 1996, there was a five-fold increase in the number of articles devoted to this topic. Why this increasing interest? Perhaps it was a natural outgrowth of the consumer movement begun in the 1960s and 1970s. Or maybe it reflected the maturation of the family medicine research agenda. Equally plausible might be the emerging competitiveness of managed care, which led to using patient satisfaction surveys to distinguish between providers. ${ }^{1}$
Though a significant number of researches have focused this topic, however, there are no universally accepted means of measuring patient satisfaction. A review of recent studies reveals some interesting findings. Satisfaction has been shown to be related directly to patient expectations; however, intuitive physician judgments about patient expectations may not correlate with true expectations. Further, patient satisfaction may not correlate with the level of clinical outcome. Recent advances have changed our understanding of this complex field. ${ }^{2}$

It is worth noting that most patient-satisfaction studies are based on patients' experiences at onetime encounters rather than their experiences over time. In addition, discussions in the literature make it clear that quality of care is not what is being measured in patient surveys. In fact, many surveys intentionally avoid asking patients how

${ }_{1}^{1}$ Professor, Department of Medicine, Rajshahi Medical College, Rajshahi.

2 Professor, Department of Pathology (Retd), Rajshahi Medical College, Rajshahi.

${ }^{3}$ Course Co-ordinator, Edward University, Dhaka Study Centre, Dhaka.

${ }^{4}$ Associate Professor, Department of Community Medicine, Dinajpur Medical College, Dinajpur. 
they feel about the quality of their care, presumably because patients are not in a position to judge their physician's technical skill. It appears that what's being measured is typically a combination of the patient's expectation before the visit, the patient's experience at the visit and the extent to which the patient experienced a resolution of the symptoms that led him or her to make the visit. ${ }^{3}$

While the literature contains a number of contradictions on the subject of patient satisfaction, it also offers a number of compelling reasons for working to improve satisfaction among our patients. Studies support the idea that patients who get better are (not surprisingly) satisfied with their care. One study, in which researchers followed up with patients three weeks after they were seen, found that most were better, but those who were still symptomatic were still worried, had unmet expectations and had lower satisfaction. ${ }^{4}$ African Americans with type-2 diabetes who were most satisfied with the helpfulness of their physicians and nurses were significantly less likely to use the emergency room. ${ }^{5}$ Patients who reported being treated with dignity and who were involved in decisions were more satisfied and more adherent to their doctor's recommendations. ${ }^{6}$ Patient satisfaction surveys of inpatient physician performance showed an inverse relationship between satisfaction and risk management episodes. ${ }^{7}$

\section{Objectives}

The main objectives of this study are:

- To estimate the indices of patient satisfaction.

- To identify and report on the perceived strengths and weaknesses of the health care services provided to the patients in hospitals/clinics.

- To provide hospitals the information about their quality improvement initiatives with respect to services provided to the patients.

- To help the management to take appropriate allocative and managerial decisions for utilization of public hospitals by people who need them most and to improve the quality of their services.

\section{Patients and method}

The survey has been conducted over 200 patients in different hospitals / clinics of Rajshahi over 6 months. Patient Satisfaction Questionnaire (PSQ) was used as the research instrument. Participants marked tick in the box (from "strongly agree" to "strongly disagree") that corresponds most closely to their response to each statement. Responses were coded 1-5 from "strongly agree" to "strongly disagree". Negatively worded questions were reverse scored (so that $1=5$, etc) so that in all cases a low score indicate satisfaction.

\section{Results}

Total 200 cases were enrolled. In the group-A 100 cases were from public hospital and in the group-B 100 cases were selected from private clinics in Rajshahi. In the group-A the mean age $( \pm S D)$ of the patients was $33.55( \pm 15.13)$ years whereas in the Group- $B$ the mean age $( \pm S D)$ of the patients was 33.17 ( \pm 16.23$)$ (Table-1). Regarding age, religion, education and marital status no significant difference were noted among the patients admitted in public and private hospitals. But there was significant differences regarding sex, occupation and monthly income among the patients admitted in public and private hospitals. More male patients were admitted in public hospital than female whereas more females were admitted in private clinics (Table-2, 3, 4, 5 and 6). Mean monthly income ( \pm SD) of the patients admitted in public hospital was Tk. 4986.00土 2385.563 whereas that of the private clinics was Tk. 5472.50 2640.28 (Table-7).

Table-1: Age

\begin{tabular}{lllll}
\hline Study site & N & $\begin{array}{l}\text { Mean } \\
\text { (Years) }\end{array}$ & $\begin{array}{l}\text { Std. } \\
\text { Deviation }\end{array}$ & Sig. \\
\hline RMCH & 100 & 33.55 & 15.125 & \\
Private Clinics & 100 & 33.17 & 16.225 & .864 \\
Total & 200 & 33.36 & 15.647 & \\
\hline
\end{tabular}

Table-2: Sex distribution

\begin{tabular}{|c|c|c|c|c|c|}
\hline & & \multicolumn{2}{|c|}{ Study site } & \multirow{2}{*}{\begin{tabular}{|l} 
Total \\
$(\%)$
\end{tabular}} & \multirow[b]{2}{*}{ Sig. } \\
\hline & & $\begin{array}{l}\text { RMCH } \\
(\%)\end{array}$ & $\begin{array}{l}\text { Private } \\
\text { Clinics (\%) } \\
\end{array}$ & & \\
\hline \multirow[t]{2}{*}{ Sex } & Male & 73 & 61 & 134 & \\
\hline & Female & 27 & 39 & 66 & .072 \\
\hline Total & & 100 & 100 & 200 & \\
\hline
\end{tabular}


Table-3: Religion

\begin{tabular}{|c|c|c|c|c|c|}
\hline & & \multicolumn{2}{|c|}{ Study site } & \multirow[t]{2}{*}{ Total (\%) } & \multirow{2}{*}{ Sig. } \\
\hline & & RMCH (\%) & Private Clinics (\%) & & \\
\hline \multirow[t]{4}{*}{ Religion } & Muslim & 87 & 89 & 176 & \\
\hline & Hindu & 9 & 11 & 20 & \\
\hline & Christian & 2 & 0 & 2 & .179 \\
\hline & Others & 2 & 0 & 2 & \\
\hline Total & & 100 & 100 & 200 & \\
\hline
\end{tabular}

Table-4: Education

\begin{tabular}{llcccc}
\hline & & \multicolumn{2}{c}{ Study site } & Total (\%) & Sig. \\
& & RMCH (\%) & Private Clinics (\%) & & 51 \\
Education & No education & 26 & 25 & 59 & \\
& Primary & 32 & 27 & 71 & .271 \\
& SSC+HSC & 34 & 37 & 19 & \\
Total & Degree+ & 8 & 11 & 200 & \\
\hline
\end{tabular}

Table-5: Occupation

\begin{tabular}{|c|c|c|c|c|c|}
\hline & & \multicolumn{2}{|c|}{ Study site } & \multirow[t]{2}{*}{ Total (\%) } & \multirow{2}{*}{ Sig. } \\
\hline & & RMCH (\%) & Private Clinics (\%) & & \\
\hline \multirow[t]{8}{*}{ Occupation } & Government service & 4 & 7 & 11 & \multirow{9}{*}{.890} \\
\hline & Non-government service & 4 & 7 & 11 & \\
\hline & Farmer & 29 & 22 & 51 & \\
\hline & Business & 14 & 15 & 29 & \\
\hline & Laborer & 8 & 4 & 12 & \\
\hline & Housewife & 20 & 27 & 47 & \\
\hline & Unemployed & 2 & 1 & 3 & \\
\hline & Others & 19 & 17 & 36 & \\
\hline Total & & 100 & 100 & 200 & \\
\hline
\end{tabular}

Table-6: Marital status

\begin{tabular}{|c|c|c|c|c|c|}
\hline & & \multicolumn{2}{|c|}{ Study site } & \multirow[b]{2}{*}{ Total (\%) } & \multirow[b]{2}{*}{ Sig. } \\
\hline & & RMCH (\%) & $\begin{array}{c}\text { Private Clinics } \\
\text { (\%) }\end{array}$ & & \\
\hline \multirow{3}{*}{$\begin{array}{l}\text { Marital } \\
\text { status }\end{array}$} & Married & 66 & 68 & 134 & \multirow{4}{*}{.793} \\
\hline & Unmarried & 34 & 30 & 64 & \\
\hline & Divorced & 0 & 2 & 2 & \\
\hline Total & & 100 & 100 & 200 & \\
\hline
\end{tabular}

Table-7: Monthly income

\begin{tabular}{lcccc}
\hline Study site & N & Mean (Taka) & Std. Deviation & Sig. \\
\hline RMCH & 100 & 4986.00 & 2385.563 & \\
Private Clinics & 100 & 5472.50 & 2640.280 & .173 \\
Total & 200 & 5229.25 & 2521.636 & \\
\hline
\end{tabular}


Table-8: Satisfaction analysis

\begin{tabular}{|c|c|c|c|c|c|}
\hline & & $\mathbf{N}$ & $\begin{array}{l}\text { Mean } \\
\text { score }\end{array}$ & Std. Error & Sig. \\
\hline \multirow[t]{3}{*}{ 1. Doctors behavior } & $\mathrm{RMCH}$ & 100 & 2.02 & .028 & \multirow{3}{*}{.529} \\
\hline & Private Clinics & 100 & 2.00 & .014 & \\
\hline & Total & 200 & 2.01 & .016 & \\
\hline \multirow{3}{*}{ 2. $\quad$ Nurses behavior } & $\mathrm{RMCH}$ & 100 & 2.12 & .052 & \multirow{3}{*}{.752} \\
\hline & Private Clinics & 100 & 2.10 & .036 & \\
\hline & Total & 200 & 2.11 & .032 & \\
\hline \multirow[t]{3}{*}{ 3. $\quad$ Cleanliness } & RMCH & 100 & 2.56 & .092 & \multirow{3}{*}{.005} \\
\hline & Private Clinics & 100 & 2.24 & .065 & \\
\hline & Total & 200 & 2.40 & .058 & \\
\hline \multirow{3}{*}{$\begin{array}{ll}\text { 4. } & \text { Environmental quietness }\end{array}$} & $\mathrm{RMCH}$ & 100 & 2.81 & .097 & \multirow{3}{*}{.000} \\
\hline & Private Clinics & 100 & 2.37 & .073 & \\
\hline & Total & 200 & 2.59 & .063 & \\
\hline \multirow[t]{3}{*}{ 5. Patients receive the best care from the staff } & $\mathrm{RMCH}$ & 100 & 2.47 & .086 & \multirow{3}{*}{.002} \\
\hline & Private Clinics & 100 & 2.16 & .049 & \\
\hline & Total & 200 & 2.32 & .050 & \\
\hline \multirow[t]{3}{*}{ 6. $\quad$ Easy to speak to my doctor } & RMCH & 100 & 3.95 & .026 & \multirow{3}{*}{.000} \\
\hline & Private Clinics & 100 & 3.40 & .091 & \\
\hline & Total & 200 & 3.68 & .051 & \\
\hline \multirow{3}{*}{ 7. $\quad$ Doctor clearly explains } & $\mathrm{RMCH}$ & 100 & 2.42 & .081 & \multirow{3}{*}{.000} \\
\hline & Private Clinics & 100 & 2.06 & .034 & \\
\hline & Total & 200 & 2.24 & .046 & \\
\hline \multirow[t]{3}{*}{ 8. $\quad$ Getting appointment at a convenient time } & $\mathrm{RMCH}$ & 100 & 2.44 & .082 & \multirow{3}{*}{.058} \\
\hline & Private Clinics & 100 & 2.24 & .065 & \\
\hline & Total & 200 & 2.34 & .053 & \\
\hline \multirow[t]{3}{*}{ 9. $\quad$ Nurses do no things carefully } & $\mathrm{RMCH}$ & 100 & 3.31 & .095 & \\
\hline & Private Clinics & 100 & 3.76 & .064 & .000 \\
\hline & Total & 200 & 3.54 & .059 & \\
\hline 10. Doctor tests to find out wrong & $\mathrm{RMCH}$ & 100 & 2.09 & .040 & \\
\hline & Private Clinics & 99 & 2.09 & .038 & .987 \\
\hline & Total & 199 & 2.09 & .028 & \\
\hline 11. Faith and confidence in the doctors & $\mathrm{RMCH}$ & 100 & 1.69 & .061 & \\
\hline & Private Clinics & 100 & 1.88 & .033 & .007 \\
\hline & Total & 200 & 1.79 & .035 & \\
\hline 12. Doctor advice over the telephone & $\mathrm{RMCH}$ & 100 & 3.93 & .033 & \\
\hline & Private Clinics & 100 & 3.38 & .093 & .000 \\
\hline & Total & 200 & 3.66 & .053 & \\
\hline 13. Doctor does not tell me enough about treatment & $\mathrm{RMCH}$ & 98 & 3.19 & .099 & \\
\hline & Private Clinics & 100 & 3.66 & .074 & .000 \\
\hline & Total & 198 & 3.43 & .064 & \\
\hline 14. Doctor explain future health & $\mathrm{RMCH}$ & 100 & 3.72 & .070 & \\
\hline & Private Clinics & 99 & 3.96 & .028 & .002 \\
\hline & Total & 199 & 3.84 & .039 & \\
\hline 15. Get advice over the telephone & $\mathrm{RMCH}$ & 100 & 3.98 & .014 & \\
\hline & Private Clinics & 99 & 3.39 & .093 & .000 \\
\hline & Total & 199 & 3.69 & .051 & \\
\hline 16. Doctor is very careful when examining & $\mathrm{RMCH}$ & 100 & 2.05 & .039 & \\
\hline & Private Clinics & 100 & 2.02 & .020 & .491 \\
\hline & Total & 200 & 2.04 & .022 & \\
\hline 17. Satisfied with hours service & $\mathrm{RMCH}$ & 100 & 2.22 & .063 & \\
\hline & Private Clinics & 100 & 2.19 & .053 & .715 \\
\hline & Total & 200 & 2.21 & .041 & \\
\hline 18. Doctor is always interested & $\mathrm{RMCH}$ & 100 & 2.09 & .035 & \\
\hline & Private Clinics & 99 & 2.04 & .025 & .249 \\
\hline & Total & 199 & 2.07 & .021 & \\
\hline 19. Good facilities for dealing with emergencies & $\mathrm{RMCH}$ & 100 & 2.14 & .038 & \\
\hline & Private Clinics & 100 & 3.11 & .049 & .000 \\
\hline & Total & 200 & 2.63 & .046 & \\
\hline
\end{tabular}




\begin{tabular}{|c|c|c|c|c|c|}
\hline \multirow[t]{3}{*}{ 20. Doctor always asks my illness } & $\mathrm{RMCH}$ & 100 & 3.72 & .070 & \multirow{3}{*}{.177} \\
\hline & Private Clinics & 100 & 3.84 & .055 & \\
\hline & Total & 200 & 3.78 & .044 & \\
\hline \multirow{3}{*}{ 21. I have not been given enough information } & $\mathrm{RMCH}$ & 100 & 3.53 & .083 & \multirow{3}{*}{.179} \\
\hline & Private Clinics & 100 & 3.68 & .074 & \\
\hline & Total & 200 & 3.61 & .056 & \\
\hline \multirow[t]{3}{*}{ 22. Satisfied with my doctor } & RMCH & 100 & 3.63 & .080 & \multirow{3}{*}{.053} \\
\hline & Private Clinics & 100 & 3.82 & .056 & \\
\hline & Total & 200 & 3.73 & .049 & \\
\hline \multirow[t]{3}{*}{ 23. Nurse does not always listen } & $\mathrm{RMCH}$ & 100 & 3.42 & .090 & \multirow{3}{*}{.011} \\
\hline & Private Clinics & 100 & 3.71 & .069 & \\
\hline & Total & 200 & 3.57 & .057 & \\
\hline \multirow[t]{3}{*}{ 24. One or two things about surgery, I am not happy } & RMCH & 2 & 3.00 & 1.000 & \multirow{3}{*}{.312} \\
\hline & Private Clinics & 100 & 3.59 & .081 & \\
\hline & Total & 102 & 3.58 & .081 & \\
\hline \multirow[t]{3}{*}{ 25. Waiting room is uncomfortable } & $\mathrm{RMCH}$ & 23 & 2.26 & .144 & \multirow{3}{*}{.049} \\
\hline & Private Clinics & 48 & 2.69 & .130 & \\
\hline & Total & 71 & 2.55 & .102 & \\
\hline \multirow[t]{3}{*}{ 26. Receptionists explain things clearly } & $\mathrm{RMCH}$ & 100 & 3.55 & .082 & \multirow{3}{*}{.001} \\
\hline & Private Clinics & 100 & 3.13 & .097 & \\
\hline & Total & 200 & 3.34 & .065 & \\
\hline \multirow[t]{3}{*}{ 27. Doctor makes me feel wasting time } & $\mathrm{RMCH}$ & 99 & 3.80 & .059 & \multirow{3}{*}{.014} \\
\hline & Private Clinics & 100 & 3.96 & .028 & \\
\hline & Total & 199 & 3.88 & .033 & \\
\hline 28. I don't feel confident discussing my problems & $\mathrm{RMCH}$ & 100 & 3.62 & .078 & \\
\hline & Private Clinics & 100 & 3.82 & .058 & .040 \\
\hline & Total & 200 & 3.72 & .049 & \\
\hline 29. Appointments are easy & $\mathrm{RMCH}$ & 100 & 2.44 & .083 & \\
\hline & Private Clinics & 100 & 2.19 & .058 & .015 \\
\hline & Total & 200 & 2.32 & .051 & \\
\hline 30. Doctor want to get rid of me & $\mathrm{RMCH}$ & 100 & 3.85 & .052 & \\
\hline & Private Clinics & 100 & 3.93 & .036 & .205 \\
\hline & Total & 200 & 3.89 & .032 & \\
\hline 31. Doctor gives me every chance to talk & $\mathrm{RMCH}$ & 100 & 2.16 & .053 & \\
\hline & Private Clinics & 100 & 2.10 & .044 & .382 \\
\hline & Total & 200 & 2.13 & .034 & \\
\hline 32. Doctor fails to appreciate how ill I am & RMCH & 100 & 3.72 & .070 & \\
\hline & Private Clinics & 99 & 3.84 & .055 & .185 \\
\hline & Total & 199 & 3.78 & .045 & \\
\hline 33. Doctor shows interest in my problems & $\mathrm{RMCH}$ & 100 & 2.14 & .051 & \\
\hline & Private Clinics & 100 & 2.12 & .046 & .771 \\
\hline & Total & 200 & 2.13 & .034 & \\
\hline 34. Receptionists ask patients the right questions & $\mathrm{RMCH}$ & 26 & 3.85 & .107 & \\
\hline & Private Clinics & 25 & 3.56 & .164 & .147 \\
\hline & Total & 51 & 3.71 & .098 & \\
\hline 35. Not enough seats in the waiting room & $\mathrm{RMCH}$ & 24 & 2.00 & .000 & \\
\hline & Private Clinics & 50 & 2.74 & .130 & .000 \\
\hline & Total & 74 & 2.50 & .097 & \\
\hline 36. Doctor does everything diagnosis & $\mathrm{RMCH}$ & 100 & 2.04 & .028 & \\
\hline & Private Clinics & 99 & 2.02 & .020 & .569 \\
\hline & Total & 199 & 2.03 & .017 & \\
\hline 37. Nurse is always very reassuring & $\mathrm{RMCH}$ & 100 & 2.41 & .081 & \\
\hline & Private Clinics & 100 & 2.18 & .052 & .017 \\
\hline & Total & 200 & 2.30 & .048 & \\
\hline 38. Doctor always puts me at case & $\mathrm{RMCH}$ & 98 & 2.14 & .050 & \\
\hline & Private Clinics & 100 & 2.04 & .024 & .065 \\
\hline & Total & 198 & 2.09 & .028 & \\
\hline 39. Waiting room seats are uncomfortable & $\mathrm{RMCH}$ & 25 & 2.16 & .111 & \\
\hline & Private Clinics & 50 & 2.68 & .129 & .011 \\
\hline & Total & 75 & 2.51 & .097 & \\
\hline
\end{tabular}




\begin{tabular}{|c|c|c|c|c|c|}
\hline \multirow[t]{3}{*}{ 40. See the doctor of my choice } & $\mathrm{RMCH}$ & 100 & 3.91 & .040 & \multirow{3}{*}{.000} \\
\hline & Private Clinics & 100 & 3.36 & .094 & \\
\hline & Total & 200 & 3.64 & .055 & \\
\hline \multirow[t]{3}{*}{ 41. Doctor is very understanding } & $\mathrm{RMCH}$ & 100 & 2.08 & .037 & \multirow{3}{*}{.412} \\
\hline & Private Clinics & 100 & 2.13 & .049 & \\
\hline & Total & 200 & 2.11 & .030 & \\
\hline \multirow[t]{3}{*}{ 42. When the doctor is busy I am examined properly } & $\mathrm{RMCH}$ & 100 & 2.15 & .052 & \multirow{3}{*}{.064} \\
\hline & Private Clinics & 100 & 2.04 & .028 & \\
\hline & Total & 200 & 2.10 & .030 & \\
\hline \multirow[t]{3}{*}{ 43. Speak to a receptionist privately } & $\mathrm{RMCH}$ & 99 & 3.67 & .070 & \multirow{3}{*}{.359} \\
\hline & Private Clinics & 100 & 3.57 & .078 & \\
\hline & Total & 199 & 3.62 & .053 & \\
\hline \multirow[t]{3}{*}{ 44. Doctor knows when tests are necessary } & $\mathrm{RMCH}$ & 100 & 2.02 & .014 & \multirow{3}{*}{.569} \\
\hline & Private Clinics & 99 & 2.01 & .010 & \\
\hline & Total & 199 & 2.02 & .009 & \\
\hline \multirow[t]{3}{*}{ 45. Nurse makes me feel wasting his/her time } & $\mathrm{RMCH}$ & 99 & 3.69 & .070 & \multirow{3}{*}{.077} \\
\hline & Private Clinics & 99 & 3.84 & .049 & \\
\hline & Total & 198 & 3.76 & .043 & \\
\hline \multirow[t]{3}{*}{ 46. Do not feel rushed when I am with doctor } & $\mathrm{RMCH}$ & 100 & 2.10 & .044 & \multirow{3}{*}{.735} \\
\hline & Private Clinics & 100 & 2.08 & .039 & \\
\hline & Total & 200 & 2.09 & .029 & \\
\hline \multirow[t]{3}{*}{ 47. Reason for medical tests } & $\mathrm{RMCH}$ & 98 & 2.76 & .098 & \multirow{3}{*}{.016} \\
\hline & Private Clinics & 100 & 2.44 & .084 & \\
\hline & Total & 198 & 2.60 & .066 & \\
\hline \multirow[t]{3}{*}{ 48. Complete medical care } & $\mathrm{RMCH}$ & 100 & 2.49 & .072 & \multirow{3}{*}{.616} \\
\hline & Private Clinics & 100 & 2.54 & .069 & \\
\hline & Total & 200 & 2.52 & .050 & \\
\hline 49. If their diagnosis is correct & $\mathrm{RMCH}$ & 100 & 3.81 & .054 & \\
\hline & Private Clinics & 100 & 3.96 & .024 & .013 \\
\hline & Total & 200 & 3.89 & .030 & \\
\hline 50. Emergency treatment & $\mathrm{RMCH}$ & 100 & 2.70 & .095 & \\
\hline & Private Clinics & 99 & 2.74 & .096 & .782 \\
\hline & Total & 199 & 2.72 & .067 & \\
\hline 51. Courteous manner & $\mathrm{RMCH}$ & 100 & 2.13 & .051 & \\
\hline & Private Clinics & 100 & 2.16 & .051 & .676 \\
\hline & Total & 200 & 2.15 & .036 & \\
\hline 52. Hurry too much when they treat me & $\mathrm{RMCH}$ & 100 & 3.22 & .098 & \\
\hline & Private Clinics & 100 & 3.01 & .100 & .135 \\
\hline & Total & 200 & 3.12 & .070 & \\
\hline 53. Doctors sometimes ignore & $\mathrm{RMCH}$ & 100 & 3.70 & .072 & \\
\hline & Private Clinics & 100 & 3.72 & .070 & .842 \\
\hline & Total & 200 & 3.71 & .050 & \\
\hline 54. Doubts about the ability of the doctor & $\mathrm{RMCH}$ & 99 & 3.91 & .032 & \\
\hline & Private Clinics & 99 & 3.99 & .010 & .018 \\
\hline & Total & 198 & 3.95 & .017 & \\
\hline 55. Doctor usually spend plenty of time with me & $\mathrm{RMCH}$ & 100 & 3.03 & .101 & \\
\hline & Private Clinics & 100 & 3.08 & .098 & .723 \\
\hline & Total & 200 & 3.06 & .070 & \\
\hline 56. Medical care right away & $\mathrm{RMCH}$ & 100 & 3.06 & .100 & \\
\hline & Private Clinics & 100 & 2.86 & .100 & .159 \\
\hline & Total & 200 & 2.96 & .071 & \\
\hline 57. Dissatisfied with some things about medical care & $\mathrm{RMCH}$ & 100 & 3.02 & .100 & \\
\hline & Private Clinics & 100 & 3.19 & .098 & .228 \\
\hline & Total & 200 & 3.11 & .070 & \\
\hline 58. Able to get medical care & $\mathrm{RMCH}$ & 100 & 3.80 & .060 & \\
\hline & Private Clinics & 100 & 3.82 & .058 & .811 \\
\hline & Total & 200 & 3.81 & .042 & \\
\hline
\end{tabular}

- Significance tests are done by one way ANOVA test. 
Regarding satisfaction parameter analysis, no significant differences were noted between public and private hospitals in relation to doctors behavior, nurses behavior, getting appointment at a convenient time, doctor tests to find out wrong, doctors examination procedure, hours of service, doctors interest, information sharing about disease, communication, receptionists behavior, nurses' attitude, emergency treatment, courteous manner and time spent by doctors with the patients. On the other hand significant differences were noted about cleanliness, environmental quietness, staff care, access to doctor, doctors explanation, nurses activity, faith and confidence in the doctors, doctor advice over the telephone, facilities for dealing with emergencies, nurses' attention, waiting room, receptionists behavior, medical tests and doctors competency (Table-8).

\section{Discussion}

Different studies have revealed that our common beliefs about medical services are: treatment seeking at public hospitals is a burden to the people in rural areas because of user charges and inadequate drug supply in hospital, doctors do not spend enough time with patients in public hospitals, drug supply in hospitals is inadequate, attendants are not allowed to accompany patients when doctors are on rounds but attendants understand \& can explain the patient's condition better, behaviors of doctors, nurses and other supporting staff are not satisfactory.

Assessment of patient satisfaction allows doctors/general practitioners to investigate the extent to which their service meets the needs of their client group ${ }^{8}$. Questionnaires that assess specific aspects of service provision will enable the practitioner to identify aspects of the service where patients are less satisfied, and potentially improve these aspects of care ${ }^{9}$. Research has shown that satisfied patients are more likely to follow treatment instructions and medical advice, probably because they are more likely to believe that treatment will be effective ${ }^{10}$. They are also less likely to change doctors and make complaints $^{11}$. It is therefore in the doctors/general practitioner's interest to know the extent of patient satisfaction with service provision ${ }^{12}$. In our present survey regarding satisfaction parameter analysis, no significant differences were noted between public and private hospitals in relation to doctors behavior, nurses behavior, getting appointment at a convenient time, doctor tests to find out wrong, doctors examination procedure, hours of service, doctors interest, information sharing about disease, communication, receptionists behavior, nurses' attitude, emergency treatment, courteous manner and time spent by doctors with the patients. But significant differences were noted about cleanliness, environmental quietness, staff care, access to doctor, doctors' explanation, nurses' activity, faith and confidence in the doctors, doctor advice over the telephone, facilities for dealing with emergencies, nurses' attention, waiting room, receptionists behavior, medical tests and doctors competency between private and public hospitals. Despite resource constraints health personnel in private health care delivery centers are providing satisfactory health services than public hospitals. And health care delivery in public hospitals should be closely monitored for further improvement to meet the demands of people.

\section{Conclusion}

Despite resource constraints health personnel in private health care delivery centers are providing satisfactory health services than public hospitals. In the face of ever increasing demand for health care services strength, weakness and future prospects of private health care facilities should be evaluated and appraised. On the other hand health care delivery in public hospitals should be closely monitored for further improvement to meet the demands of people.

\section{Acknowledgement}

We would like to thank Dr. Mamun and Dr. Abdullah, Honorary Medical Officer, MU-I, Rajshahi Medical College Hospital for collection of data for this study. 


\section{References}

1. White B. Measuring patient satisfaction: how to do it and why to bother? Fam Pract Manag. 1999; 6(1):40-4. 2.1.

2. Nettleman. Patient satisfaction--what's new? Clin Perform Qual Health Care. 1998; 6(1):33-7.

3. Thiedke C. What Do We Really Know About Patient Satisfaction? Fam Pract Manag. January 2007, Vol. 14, No. 1, pages 33-6.

4. Kroenke K, Jackson JL. Outcome in general medical patients presenting with common symptoms. Fam Pract. 1998; 15:398-403.

5. Gary TL, Maiese EM, Batts-Turner M, Wang NY, Brancati FL. Patient satisfaction, preventive services and emergency room use among African-Americans with type-2 diabetes. Dis Manag. 2005; 8:361-371.

6. Beach MC, Sugarman J, Johnson RL, Arbelaez JJ, Duggan PS, Cooper LA. Do patients treated with dignity report higher satisfaction, adherence and receipt of preventive care? Ann Fam Med. 2005; 3:331-338.
7. Stelfox HT, Gandhi TK, Orav EJ, Gustafson ML. The relation of patient satisfaction with complaints against physicians and malpractice lawsuits. Am J Med. 2005; 118:1126-1133.

8. Avis M, Bond M, Arthur A. Satisfying solutions? A review of some unresolved issues in the measurement of patient satisfaction. J Advan Nurs 1995; 22:316-22.

9. Harris LE, Swindle RW, Mungai SM, et al. Measuring patient satisfaction for quality improvement. Med Care 1999; 37:1207-13.

10. Hardy GE, West MA, Hill F. Components and predictors of patient satisfaction. $\mathrm{Br} \mathrm{J}$ Health Psychol 1996; 1:65-85.

11. Taylor SE. Health psychology. New York: McGraw Hill, 1995.

12. Woodward CA, Ostbye T, Craighead J, et al. Patient satisfaction as an indicator of quality care in independent health facilities: developing and assessing a tool to enhance public accountability. Am J Med Qual 2000; 15:94-105. 